\title{
Bringing Scenario Planning Home to KU
}

\section{Jennifer Church-Duran, Assistant Dean for User Services, University of Kansas Libraries Deborah Ludwig, Assistant Dean for of Collections \& Scholar Services, University of Kansas Libraries}

nergized by the immersive learning process at the ARL scenario-planning workshop in March 2011, the University of Kansas (KU) Libraries decided to introduce scenario planning to library staff later that spring, to support development of a new strategic plan. From the onset, using scenario planning as an effective strategic tool required comprehensive preparation. The ARL 2030 Scenarios User's Guide ${ }^{1}$ and related materials served as a valuable template, but the process necessitated adaptation for our organizational climate. Joined by a team of energized staff with organizational development and leadership interests, we prepared and conducted two workshops aimed at broad staff participation. We reaped many benefits from the process, gaining valuable insights into the purpose and practice of scenario planning. We also met with a few unexpected challenges.

\section{Process}

To kick off the project, we hosted several 90-minute brown-bag orientations for all interested library faculty and staff. For most staff, scenario planning represented an entirely new model for strategic thinking. As a result, the sessions offered a much-needed conceptual overview and the opportunity to learn more, before committing to the extensive planning workshops that followed. We were delighted at the turnout for these preliminary meetings and quickly capitalized on staff interest by offering two full workshops, one held in May and another in July, with 43 participants total.

Time was a key factor in rolling out these workshops. To balance our operational responsibilities with the opportunity for this strategic exploration, we modified the ARL proposed agenda of two full days. Instead, we developed a half-day/full-day/half-day schedule. This adaptation proved to be doubly beneficial, generating increased flexibility for attendance, while giving participants a chance to decompress overnight and return to the process refreshed the next morning.

The conversation generated during the preliminary brown-bag sessions helped set the stage for more detailed planning. The questions raised by staff illustrated both the advantages and challenges of implementing a set of scenarios that were constructed without direct engagement by local participants. The benefits were simple but substantial. In developing these narratives at the national level, ARL effectively streamlined the workflow and greatly reduced the time commitment needed for local implementation. Additionally, the resulting scenarios contained unique insights drawn from expertise across the profession and throughout higher education.

However, there were also challenges created by disconnecting scenario building from scenario planning. Constructing scenarios requires substantial intellectual engagement and a thorough analysis of trends and driving forces. It starts to frame the "outside-in" thinking at the core of scenario planning which, in turn, fosters a sense of ownership for the narratives when used in the later development of scenario strategies. In the absence of this foundational intellectual investment, KU Libraries' participants were responding to the product of someone else's thinking. This created a larger credibility gap-an increased questioning of the validity of the futures described and less initial conviction in responding to the scenarios. 
Skillful facilitation and preparation helped bridge this gap and advanced the viability of the scenarios. At KU, we worked to build a foundation of understanding by pre-assigning staff to a specific scenario and working group and requesting that participants undertake advanced and in-depth study of the narratives. However, the most effective preparation for staff was a pre-workshop assignment to research and uncover early indicators that lent plausibility to each scenario. Staff explored the current literature and carried out a brief climate study, gathering evidence of future scenario characteristics already at play in today's environment. This early-indicator work brought in the driving forces and current trends that originally shaped the scenarios, generating stronger "buy-in" and closing the credibility gap. It also demonstrated the interrelated dynamics woven through the scenario set, as some indicators apply across multiple scenarios.

The KU workshops were launched by sharing the early-indicator research. The resulting conversations continued to reinforce the viability of the scenarios as planning tools. This transitioned to a "deep-dive" activity: small group exploration and analysis of the assigned scenario. The resulting analysis proved quite successful in brainstorming and developing strategies that would work well within that one possible future represented by the assigned scenario.

The last, crucial step was the more challenging: the transition from small-group, deep-dive analysis of a single scenario to the use of the scenarios as a comprehensive set. The purpose of this final stage was to identify actions that could work across all possible futures and strategically place KU Libraries in a highly flexible, adaptable position when change occurs. The outcome is a strategic agenda robust enough to align present action to a broad range of future challenges and opportunities.

As the ARL 2030 Scenarios User's Guide notes, a successful agenda is shaped by these key characteristics:

- Robust-works across a divergent and challenging set of scenarios.

- Distinctive and Advantaged-leverages the distinctive competencies of the organization toward achieving an advantaged position in the external research environment.

- Inspiring-delights the end-use customer and inspires the staff.

- Focused-requires strategic choices of things to do and things not to do, assessed against a realistic understanding of capabilities and resources. ${ }^{2}$

In the KU workshops, both the participants and the facilitators struggled with the development of a robust strategy. During the deep-dive process, each working group developed a rich array of possibilities to position the libraries for the potential that existed within the assigned future. This process, while incredibly valuable, tended to lock participants into thinking centered primarily on one scenario. Moving into the creation of a robust agenda required searching for points of overlap and mutual benefit that support all potential futures.

Stretching the conversation to a broader view of interlinked futures demanded substantial intellectual work. During the phase of "Identifying Robust Strategic Options," the participants in both workshops experienced confusion and uncertainty on what was being asked of them and the intended final outcomes of this stage. This frustration was increased by how differently the groups organized their content from the earlier deep-dive analysis. There was significant variance in the level of detail each 
group provided for their respective scenario's strategic options and in how their input was described on the corresponding Post-its. As a result, when the larger group worked with these materials to identify broad strategic options, it was challenging to make reasonable comparisons or determine points of connections.

These challenges eventually ground efforts to a complete halt. The facilitators took this opportunity to stop the process and open up a frank discussion on what alternatives in workshop process might make more sense to the group and move us forward productively. Real-time recommendations evolved, based on the materials we had available and the needs revealed while attempting to work with these pieces. Based on all suggestions from workshop participants and facilitators, we experimented with other workflows not specifically outlined in the workshop guide. This included a question-and-answer period for groups to obtain further information from one another and use of a round-robin reporting technique.

The round-robin process was particularly effective. Prior to implementing it, each group presented a comprehensive reporting of its own robust strategic options. There was often considerable overlap of ideas across groups and it was time-consuming to hear the same information reported over and over again. With the round-robin technique, we moved quickly from group to group, each time contributing one new option that was not currently on the board. The result was a faster compilation of ideas without unnecessary duplication. Eventually, through this type of small- and large-group interaction, the attendees pulled together a set of strategies with the potential to work across the envisioned set of future possibilities.

\section{Outcomes}

When the ARL scenario-planning project was initiated, we envisioned a relatively seamless movement from scenario-planning outcomes to the development of KU Libraries' next strategic plan. The university, however, launched a comprehensive campus strategic planning process during the same time period. In an effort to align the libraries closely with the campus vision and direction, we delayed further local action after the workshops. Our goal was to delay library planning until we had a thorough knowledge and understanding KU's future directions. Fortunately, the libraries sought and achieved considerable opportunity to participate in the campus planning activities, and we were able to bring insight from the scenario project to the broader campus-wide discussion.

As a result, we did not lose the benefits of the scenario-exploration efforts while waiting for the campus plan, titled Bold Aspirations, ${ }^{3}$ to unfold. The strategic thinking associated with analyzing the scenarios, and the in-depth exploration of alternative futures, influenced our contributions to the campus plan. It also created an ideal foundation for the libraries' own strategic-planning development that would soon follow. The power of the scenario exercise existed in the transformational nature of the process, not in achievement of a final product.

When the completed KU plan was announced, the libraries chose to model their own strategicplanning efforts directly on the campus process. This included a Strategic Planning Steering Committee with broad, library-wide representation. The committee oversaw and coordinated the efforts of multiple working groups. The committee also drew heavily on the goals and strategies from the Bold Aspirations document, in order to shape the focus of the libraries' planning work. 
Many of the scenario workshop participants subsequently served on the libraries' strategic-planning groups. As the Steering Committee began conducting an environmental scan with a detailed SWOT analysis, the earlier scenario activities appeared to have both informed thinking and enhanced our ability to de-personalize discussions about the future. Engagement with scenario-planning concepts seemed to create a mindset regarding informed decision making as a tool for adaptive foresight. This, in turn, allowed staff to consider how the future could be seen more clearly from our anchor within present reality. The influence of the scenario project was demonstrated by the surprising connections that emerged between the scenario workshop outcomes and KU Libraries' new strategic goals that form the foundation of the libraries' five-year plan, which is currently under development.

The robust agenda that emerged from our first workshops included the following strategies:

- Become an agile organization able to rapidly adapt and change.

- Develop new and diverse funding models.

- Create a culture of collaboration and partnership for a broader audience.

- Rethink and realign resources, collections \& infrastructure.

Two of the thematic goals from our developing strategic plan directly reflect these workshop strategies:

- Strengthen KU Libraries' position as an agile, responsive organization capable of continual improvement and change.

- Stabilize and grow existing funding sources, secure new funding opportunities, and enhance public accountability.

Specific actions under the robust strategies are also reflected in the current strategic planning process. The third scenario strategy, "Create a culture of collaboration and partnership for a broader audience," included activities focused on learning more about KU researchers and understanding their needs, strengthening partnerships that support research in areas such as publishing and data management and curation, and maximizing support for interdisciplinary research. All of these activities are finding their way into our current strategic planning exercise as well as into our operational work at KU Libraries today.

We invested a considerable amount of time preparing for the scenario workshops to ensure a positive outcome and that work was worthwhile. A majority of the participants rated the workshops as "excellent" or "very good." All participants responded that the balance between presentations by the workshop leaders and group discussion and activities represented a good ratio.

Overall, most participants found the workshops were effective in helping them understand the existing and future directions of KU Libraries. By shifting strategic thinking from a reactive to a proactive stance, and from an internal to an external focus, we are better equipped to deal with a future driven by regional, national, and world events beyond our control. As facilitators for the scenario workshops, we feel the time that we invested in preparing and conducting these workshops was well spent and has been instrumental in helping KU Libraries create its new strategic plan to meet the challenges ahead. 
1 Association of Research Libraries and Stratus, Inc., The ARL 2030 Scenarios: A User's Guide for Research Libraries (Washington, DC: ARL, 2010), http://www.arl.org/bm doc/arl-2030-scenariosusers-guide.pdf.

2 ARL and Stratus, ARL 2030 Scenarios, 42.

3 University of Kansas (KU), Bold Aspirations: The Strategic Plan for the University of Kansas, 2012-2017 (Lawrence, Kansas: KU Office of the Provost, 2011), http://www.provost.ku.edu/planning/.

(C) 2012 Jennifer Church-Duran and Deborah Ludwig

\section{(c) (1) (3) (2) \\ BY NC SA}

This article is licensed under a Creative Commons Attribution-Noncommercial-Share Alike 3.0 United States License. To view a copy of this license, visit http://creativecommons.org/licenses/by-nc-sa/3.0/us/.

To cite this article: Jennifer Church-Duran and Deborah Ludwig. "Bringing Scenario Planning Home to KU." Research Library Issues: A Quarterly Report from ARL, CNI, and SPARC, no. 278 (March 2012): 12-16. http://publications.arl.org/rli278/. 